\title{
Experimental phasing at low energy at EMBL beamline P13
}

Guillaume Pompidor $^{1}$, Gleb Bourenkov ${ }^{1}$, Isabel Bento ${ }^{1}$, Johanna Hakanpää ${ }^{1}$, Ivars Karpics ${ }^{1}$, Thomas Schneider $^{1}$

${ }^{1}$ European Molecular Biology Laboratory, Hamburg, Germany

E-mail: pompidor@embl-hamburg.de

The P13 Macromolecular Crystallography beamline, operated by the EMBL at Petra III, enables diffraction data collection over a wide energy range, from $4 \mathrm{keV}$ to $17.5 \mathrm{keV}(0.7-3.1 \AA)[1]$. In order to fully exploit the beamline capability at low energy (1012 ph.s-1 at $5 \mathrm{keV}$ ), the PILATUS 6M-F detector, with a $450 \mu \mathrm{m}$ Si sensor thickness and with a custom calibration at low energy, is mounted on a $2 \theta$-angle stage to increase the maximum resolution achievable $(2.4 \AA$ at $4 \mathrm{keV})$.

We will present and describe the typical experimental setup for long-wavelength data collection. For low-energy experiments, typically below $5.5 \mathrm{keV}$, the air absorption can be drastically reduced by the use of a helium path. The gas is circulating in a homemade cone, which can be fixed on the detector upon user request. In this configuration, at the minimal crystal to detector distance $(135 \mathrm{~mm})$, only the first $30 \mathrm{~mm}$ of the path of the diffracted beams is in air.

The beamline can be configured for low-energy data collection in about 10 minutes and remains fully compatible with the use of the MARVIN sample changer for high-throughput screening, standard cryogenic cooling, standard SPINE pins for sample mounting, and the mini-K of the MD2 diffractometer allowing the reorientation of the crystal for anomalous strategic data collection.

To illustrate the beamline capabilities, several examples of structure determination using intrinsic anomalous scatterers, i-e sulfur SAD or calcium (MAD at the Ca $\mathrm{K}$ edge at $4.05 \mathrm{keV}$ ) will be presented. In particular, we will demonstrate the efficiency of the He-path in terms of improvement of diffraction data quality.

To extend the experimental opportunities, a small laboratory dedicated to the preparation of derivative is available to users close to the beamlines. With more than 150 compounds available, the heavy atom library contains many elements (Cd, Pd, U...) exhibiting strong anomalous signal at low energy, which are excellent candidates for SAD and MAD experiment at long wavelength.

The EMBL Integrated Facility for Structural Biology in Hamburg provides services for high-throughput crystallization. Crystals can be harvested with a Crystal Direct Harvester system.

For beamtime: http://smis.embl-hamburg.de

For services: http://www.embl-hamburg.de/services

Cianci, M. et al., (2017). Journal of synchrotron radiation 24, 323-332.

Keywords: Long-wavelength phasing 